\title{
The Chemtrak Hp Chek Fingerstick Whole Blood Serology Test for the Detection of Helicobacter pylori Infection
}

\author{
W. D. Chey, M.D., U. K. Murthy, M.D., W. Linscheer, M.D., C. Barish, M.D., D. Riff, M.D., H. Rubin, M.D., \\ M. Safdi, M.D., H. Schwartz, M.D., U. Shah, M.D., L. Wruble, M.D., and H. M. T. El-Zimaity, M.D. \\ University of Michigan, Ann Arbor, Michigan; VA Medical Center, Syracuse, New York; Raleigh, North Carolina; Anaheim, \\ California; Beverly Hills, California; Cincinnati, Ohio; Miami, Florida; Leonardstown, Maryland; Memphis, Tennessee; \\ and VA Medical Center, Houston, Texas
}

Objective: To evaluate a new whole blood serology test (Hp Chek; ChemTrak) that detects IgG antibodies to Helicobacter pylori. Methods: The study was conducted at 10 sites within the United States. Patients undergoing upper endoscopy for dyspepsia were recruited for enrollment. Those treated for $H$. pylori infection within a year of endoscopy and those who had regularly used proton pump inhibitors, bismuth compounds, or antibiotics within a month of endoscopy were not eligible. During endoscopy, specimens were obtained from the corpus and antrum for histological examination, which was performed by a single experienced pathologist. The Hp Chek was tested using whole blood and serum. Serum was also tested with a reference enzyme-linked immunosorbent assay (ELISA) at a centralized location. Test characteristics for the Hp Chek and ELISA were calculated using histology as the "gold standard." Results: Two hundred eighty-seven patients (140 women and 147 men; mean age $53 \pm 6 \mathrm{yr}$ ) were enrolled. The $\mathrm{Hp}$ Chek was easy to perform and yielded results $9 \mathrm{~min}$ after inoculation of the test cassette with whole blood or serum. When the Hp Chek used with whole blood was compared with histology as the gold standard, the sensitivity was $88 \%$, specificity $85 \%$, positive predictive value $83 \%$, negative predictive value $90 \%$, and percent agreement $86 \%$. There were no statistically significant differences among the results obtained with the $\mathrm{Hp}$ Chek using whole blood, the Hp Chek using serum, or reference ELISA. Conclusions: The Hp Chek whole blood serology test was easy to perform and rapid and yielded performance characteristics comparable to those of a reference ELISA or the Hp Chek used with serum. (Am J Gastroenterol 1998;93:16-19. (1998 by Am. Coll. of Gastroenterology)

\section{INTRODUCTION}

Diagnostic tests for Helicobacter pylori can be divided into those that require endoscopy with gastric mucosal bi-

Received May 20, 1997; accepted Aug. 24, 1997. opsy (rapid urease testing, histology, culture) and those that can be performed independently of endoscopy (serology, urea breath testing) (1). Serology is currently the most cost-effective means of testing for $H$. pylori infection (1). The role of $H$. pylori infection in the pathogenesis of dyspeptic symptoms in patients with no evidence of peptic ulcer disease remains poorly defined (2). However, preliminary work using decision analytical modeling suggested that it may be cost-effective to treat patients for $H$. pylori infection without evaluation with upper endoscopy (3). For this reason, it is anticipated that serology testing will become increasingly important in the management of dyspeptic patients, particularly in the primary care setting (4).

The majority of currently available serology tests for $H$. pylori rely upon the identification of specific immunoglobulin $\mathrm{G}(\mathrm{IgG})$ antibodies that can be found in saliva (5) and blood (6). Tests for the serodiagnosis of $H$. pylori in blood can he classified as quantitative (enzyme-linked immunosorbent assay [ELISA]) or qualitative. A number of qualitative tests that are easy to perform and inexpensive are now commercially available $(7,8)$. These tests were originally developed for use with serum, but recently, several companies have introduced products intended for use with whole blood. Unfortunately, the sensitivity of the current generation of whole blood serology tests has been inconsistent $(9-12)$.

We conducted a multicenter trial to evaluate the test characteristics of a new whole blood serology test manufactured by ChemTrak (Hp Chek, formerly the "Accumeter"; Sunnyvale, CA). Results obtained with the Hp Chek were compared with histology and with a reference ELISA.

\section{MATERIALS AND METHODS}

\section{Patient population}

This study was conducted at 10 geographically diverse sites in the United States. Study sites included seven community gastroenterologists' offices, two university medical centers, and one VA Medical Center. Patients undergoing upper endoscopy for dyspeptic symptoms were recruited for enrollment. Those who had received treatment for $H$. pylori 
infection within a year of endoscopy were not eligible for this trial. Therapy with proton pump inhibitors, bismuth compounds, metronidazole, amoxicillin, or tetracycline within 1 month of upper endoscopy also excluded patients from enrollment. Patients who had undergone previous gastric surgery, except oversewing of a perforation, were not enrolled in this study. All participants provided written informed consent. This protocol was approved by the Institutional Review Board at each participating hospital.

\section{Study protocol}

At the time of upper endoscopy, all study patients underwent gastric mucosal biopsy for histological examination (two corpus and two antrum). Biopsy specimens were placed in formalin and transported to a centralized location (Houston VA Medical Center, Texas) for histological evaluation by a single experienced pathologist using the Genta stain (13).

Immediately after upper endoscopy, the patients underwent whole blood serology testing with the Hp Chek test cassette. For this test, a drop of blood obtained by fingerstick was placed directly into the sample well of the test cassette. As sample flowed through the blood separation device in the cassette, red cells were selectively removed. Approximately $1 \mathrm{~min}$ after administration of the blood sample, the cassette tab was pulled, initiating the test procedure. Over the next $9 \mathrm{~min}$, a colored front migrated up the testing window, followed by a clear front of wicking reagent. Each test cassette also had an internal positive control containing immobilized human IgG antibodies to $H$. pylori. A positive control indicated that the test cassette operated properly. Test results were read visually and interpreted by the presence of one red line (positive control, negative test = negative study) or two red lines (positive control, positive test $=$ positive study) in the testing window. Operators reported that the test was easy to perform and interpret.

In addition to the fingerstick whole blood serology test. the patients donated $15 \mathrm{ml}$ of whole blood by standard venipuncture. This whole blood sample was centrifuged promptly, and a drop of serum was tested with the Hp Chek using a protocol similar to that described above.

Another aliquot of serum was frozen and sent to the clinical laboratory at ChemTrak (Sunnyvalc. CA) for testing with a reference ELISA (HM-CAP; Enteric Products Inc. Stony Brook, NY). Reference ELISA testing was performed according to the manufacturer's package insert by an experienced microbiologist.

\section{Interpretation of data and statistical analysis}

Sensitivity (true positive [TP]/TP + false negative [FN]), speciticity (true negative [TN]/TN + false positive [FP]). positive predictive value (PPV; TP/TP + FP), negative predictive value (NPV; TN/TN + FN), and percent agreement were determined for the Hp Chek with whole blood and serum and for the reference ELISA using histology as the "gold standard." Test characteristics were also deter-
TABLE 1

Performance Characteristics of the Hp Chek With Whole Blood or Serum and a Reference ELISA Using Histology as the Gold Standard

\begin{tabular}{lccccc}
\hline & Sensitivity & Specificity & PPV & NPV & $\%$ Agreement \\
\hline $\begin{array}{l}\text { Hp Chek } \\
\quad \text { Whole blood) }\end{array}$ & $88 \%$ & $85 \%$ & $83 \%$ & $90 \%$ & $86 \%$ \\
$\begin{array}{l}\text { HP Chek } \\
\quad(\text { Serum) }\end{array}$ & $88 \%$ & $84 \%$ & $82 \%$ & $89 \%$ & $86 \%$ \\
ELISA* & $92 \%$ & $79 \%$ & $79 \%$ & $92 \%$ & $85 \%$ \\
\hline
\end{tabular}

Sample consists of 287 patients undergoing upper endoscopy for dyspeptic symptoms. PPV $=$ positive predictive value; $\mathrm{NPV}=$ negative predictive value; $\%$ agreement $=$ percentage of patients who had the same H. pylori status as established by the Hp Chek or ELISA and histology.

* HM-CAP: Enteric Products Inc., Stony Brook. NY.

mined for the Hp Chek with whole blood and serum using the reference ELISA as a basis for comparison. Finally, test characteristics are also presented for the Hp Chek using whole blood when discrepent histology results were resolved with the reference ELISA. Test characteristics were compared for statistically significant differences using a 2-way analysis of variance.

\section{RESULTS}

\section{Patient characteristics}

Two hundred eighty-seven patients (140 women and 147 men; mean age $53 \pm 6 \mathrm{yr}$ ) enrolled in this trial. The study population was $72 \%$ white, $14 \%$ Hispanic, $11 \%$ African American. $2 \%$ Asian, and $1 \%$ other. Histological evidence of $H$. pylori infection was found in 131 patients $(46 \%)$.

\section{Hp Chek test characteristics}

When the Hp Chek used with whole blood was compared with histology as a gold standard, the sensitivity was $88 \%$. specificity $85 \%$, PPV $83 \%$, NPV $90 \%$, and percent agreement $86 \%$ (Table 1). The Hp Chek with serum produced similar values for sensitivity, specificity, PPV, NPV, and percent agreement of $88 \%, 84 \%, 82 \%, 89 \%$, and $86 \%$, respectively. Corresponding values for sensitivity, specificity. PPV, NPV, and percent agreement with the reference ELISA using histology as a gold standard were $92 \%, 79 \%$, $79 \%, 92 \%$, and $85 \%$. There were no statistically significant differences among the test characteristics obtained with the Hp Chek using whole blood, the Hp Chek using serum, or the reference ELISA.

When the reference ELISA was used as the gold standard, the Hp Chek performed with whole blood had a sensitivity of $87 \%$, specificity of $95 \%$, PPV of $95 \%$, NPV of $87 \%$, and overall agreement of $91 \%$. Test characteristics of the Hp Chek using whole blood and serum relative to the reference ELISA are presented in Table 2. Agreement between Hp Chek results obtained from whole blood versus serum was $97 \%$.

When discrepant results with histology were resolved using ELISA, test characteristics of the Hp Chek using whole blood improved to a sensitivity of $93 \%$, specificity of 
TABLE 2

Performance Characteristics of the Hp Chek With Whole Blood or Serum Using Reference ELISA as a Basis for Comparison

\begin{tabular}{lccccc}
\hline & Sensitivity & Specificity & PPV & NPV & $\%$ Agreement \\
\hline $\begin{array}{l}\text { Hp Chek } \\
\quad \text { (Whole blood) }\end{array}$ & $87 \%$ & $95 \%$ & $95 \%$ & $87 \%$ & $91 \%$ \\
$\begin{array}{l}\text { Hp Chek } \\
\text { (Serum) }\end{array}$ & $87 \%$ & $.95 \%$ & $95 \%$ & $87 \%$ & $91 \%$ \\
\hline
\end{tabular}

Sample consists of 287 patients undergoing upper endoscopy for dyspeptic symptoms. $\mathrm{PPV}=$ positive predictive value; $\mathrm{NPV}=$ negative predictive value: $\%$ agreement $=$ percentage of patients who had the same H. pylori status as established by the Hp Chek and ELISA.

$98 \%$, PPV of $98 \%$, NPV of $93 \%$, and overall agreement of $95 \%$.

\section{DISCUSSION}

In this age of cost consciousness and practice guidelines, the management of patients with dyspeptic symptoms and no warning signs (such as advanced age, weight loss, evidence of bleeding or anemia, or progressive, unrelenting symptoms) continues to be a topic of heated debate. Using decision analytical modeling, some investigators have suggested that it would be most cost-effective to treat this population empirically for $H$. pylori infection (3). However, it is clear that such a strategy would lead to the unnecessary treatment of the majority of dyspeptic patients, as $<50 \%$ are infected with the organism $(14,15)$. In addition, some of the presumptions necessary for such decision analytical modeling were made before the availability of inexpensive, officebased serology kits to detect $H$. pylori infection. The introduction of such tests could have a profound influence on the cost-benefit ratio of identifying infected patients before initiating therapy, particularly in the primary care setting.

Recently, several manufacturers have introduced rapid, office-based serology tests that can be performed using whole blood. There is no dispute regarding the convenience of these tests. However, the published literature addressing the performance characteristics of these tests has yielded inconsistent results $(9-12)$. We report the test characteristics of a new fingerstick whole blood serology test, the ChemTrak Hp Chek. The Hp Chek relies upon the principle of immunochromatography to identify specific IgG antibodies to $H$. pylori. The Hp Chek proved easy to perform and yielded a positive or negative result within $10 \mathrm{~min}$ of inoculation with whole blood or serum.

Using histology as a basis for comparison, the Hp Chek yielded a sensitivity, specificity, PPV, and NPV comparable to those of a reference ELISA. Test characteristics of the Hp Chek were at least equivalent and arguably superior to those of other rapid, whole blood serology tests evaluated in recent studies $(9-12)$. Agreement between results obtained with the Hp Chek using whole blood versus serum was $97 \%$, suggesting that these two means of performing the test are interchangeable. In addition, we believe that the ethnic and geographical diversity of the patient population studied makes our observations that much more credible. Given the heterogeneity in $H$. pylori strains (16) and differences in the prevalence of infection among patient populations (17), one wonders whether a lack of ethnic and geographical diversity may explain, at least in part, the discrepant results found in previous studies evaluating other whole blood serology tests.

We used histology as the gold standard for this protocol. This method of identifying $H$. pylori infection is certainly not infallible. It is clear that the histological diagnosis of $H$. pylori infection can be negatively influenced by a lack of experience on the part of the interpreting pathologist, the recent use of various drugs (including antibiotics, bismuthcontaining compounds, and proton pump inhibitors), and the protocol used to obtain gastric mucosal biopsy specimens (1). We attempted to address each of these variables in the study protocol. First, histological examination was performed by a single experienced pathologist using the Genta stain (13). Second, we excluded patients treated for $H$. pylori within a year of endoscopy and those recently treated with antibiotics or proton pump inhibitors. Finally, we used a standardized biopsy protocol, which included obtaining two biopsy specimens from both the corpus and antrum in each patient at all study sites. Given these precautions, the literature suggests that the sensitivity and specificity of histology would approach or exceed $95 \%(1,18,19)$.

In conclusion, the Hp Chek is a rapid, easy-to-perform serology test that can be used to examine whole blood or serum for the presence of IgG antibodies to $H$. pylori. The Hp Chek achieved test characteristics similar to those observed with a reference ELISA. This test should provide an accurate means of screening symptomatic patients for $H$. pylori infection in the office setting.

\section{ACKNOWLEDGMENTS}

This study was supported by ChemTrak and Astra-Merck. We appreciate the contributions of J. Barnett, G. H. Elta, T. Nostrant, W. L. Hasler, and J. Scheiman at the University of Michigan Medical Center in Ann Arbor, Michigan, and R. Rapier at the University of California at San Diego to this protocol.

Reprint requests and correspondence: W. D. Chey, M.D., 3912 Taubman Center, Box 0362. Ann Arbor, MI 48109-0362.

\section{REFERENCES}

1. Chey WD. The diagnosis of Helicobacter pylori. Hosp Physician 1997;33:11-22.

2. Talley NJ. A critique of therapeutic trials in Helicobacter pyloripositive functional dyspepsia. Gastroenterology 1994;106:1174-83.

3. Fendrick AM, Chernew ME, Hirth RA, et al. Alternative management strategies for patients with suspected peptic ulcer disease. Ann Intern Med 1995:123:260-8.

4. Ofman JJ, Etchason J. Fullerton S, et al. Management strategies for Helicobacter pylori-seropositive patients with dyspepsia: Clinical and economic consequences. Ann Intern Med 1997;126:280-91. 
5. Luzza F, Maletta M, Imeneo M. et al. Salivary-specific immunoglobulin $\mathrm{G}$ in the diagnosis of Helicobacter pvlori infection in dyspeptic patients. Am J Gastroenterol 1995;90:1820-3.

6. Evans DJ, Evans DG, Gralıam DY, et al. A sensitive and specific serologic test for detection of Campylobacter pylori infection. Gastroenterology 1989;96:1004-8.

7. Graham DY, Evans DJ, Peacock J, et al. Comparison of rapid serological tests (FlexSure HP and QuickVue) with conventional ELISA for detection of Helicobacter pylori infection. Am J Gastroenterol 1996:91:942-8.

8. Chey WD, Murthy UK, Linscheer WG, et al. A comparison between 4 different commercially available serology tests for Helicobacter pvlori. Gastroenterology 1996:110:A80 (abstract).

9. Borody TJ. Andrews P. Shortis NP. Evaluation of whole blood antibody kit to detect active Helicobacter pvlori infection. Am J Gastroenterol 1996:91:2509-12.

10. Lahaie RG. Ricard N. Validation of Helisal whole blood, serum, and saliva tests for the non-invasive diagnosis of $H$. pylori infection. Gastroenterology 1996:110:A167 (abstract).

11. Sadowski D. Cohen H, Laine L, et al. Evaluation of the FlexSure HP fingerstick blood test for the detection of $H$. pylori infection. Gastroenterology 1996;110:A246 (abstract).

12. Cohen H, Retama B, Johnson C. Evaluation of a rapid test to detect IgG antibodies to Helicobacter pylori using fingerstick whole blood samples. Gastroenterology 1996;110:A83 (abstract).

13. Genta RM, Robason GO, Graham DY. Simultaneous visualization of Helicobacter pylori and gastric morphology: A new stain. Hum Pathol 1994;25:221-6.

14. Elta GH, Scheiman JM, Barnett JL, et al. Long-term follow-up of Helicobacter pylori treatment in non-ulcer dyspepsia patients. Am J Gastroenterol 1995;90:1089-93.

15. Greenberg RE, Bank S. The prevalence of Helicobacter pylori in non-ulcer dyspepsia. Arch Intern Med 1990;150:2053-5.

16. Megraud F. H. pylori species heterogeneity. In: Hunt RH, Tytgat GNJ, eds. Helicobacter pylori: Basic mechanisms to clinical cure. Hingham, MA: Kluwer Academic Publishers, 1994:28-40.

17. Loy CT, Irwig SM, Katelaris PH, et al. Do commercial serological kits for Helicobacter pylori infection differ in accuracy? A meta-analysis. Am J Gastroenterol 1996;91:1138-44.

18. Cutler AF, Havstad S, Ma CK, et al. Accuracy of invasive and noninvasive tests to diagnose Helicobacter pylori infection. Gastroenterology 1995;109:136-41.

19. Thijs JC, van Zwet AA, Thijs WJ, et al. Diagnostic tests for Helicobacter pylori: A prospective evaluation of their accuracy, without selecting a single test as the gold standard. Am J Gastroenterol 1996; 91:2125-9. 